\title{
LAS NUEVAS TECNOLOGÍAS COMO HERRAMIENTA PARA LA IGUALDAD DE GÉNERO: MUJERES INMIGRANTES RELIGIOSAS DEL BARRIO DEL RAVAL, BARCELONA
}

\author{
Josep Lluís Micó Sanz \\ Míriam Díez Bosch \\ joseplluisms@blanquerna.url.edu \\ miriamdb@blanquerna.url.edu \\ Alba Sabaté Gauxachs \\ albasg@blanquerna.url.edu \\ Observatori Blanquerna de Comunicació, Religió i Cultura - España
}

Recibido: 29-02-2016

Aceptado: $18-05-2016$

\section{Resumen}

En el barrio del Raval (Barcelona) viven más de 10.000 mujeres inmigrantes. Traen consigo tradiciones y religiones que se incorporan al variopinto abanico que convive en la citada zona de la ciudad. En este estudio hemos investigado el papel de las nuevas tecnologías en el entorno de las mujeres inmigrantes de distintas religiones y países. Nuestro objetivo es saber si estas herramientas suponen un elemento para la igualdad de género y la integración. Para ello hemos realizado 238 entrevistas que nos han permitido obtener datos significativos. Tras las conversaciones, nos damos cuenta de que existe un elevado acceso a las tecnologías pero que a pesar de su potencial integrador, el uso que le dan se limita a su entorno más próximo y, en cualquier caso, potencia más la relación con el país de origen y el pasado que la integración con el nuevo contexto y el presente.

Palabras Clave: Mujer, igualdad, religión, tecnología, redes sociales, inmigración, género.

\begin{abstract}
There are more than 10,000 immigrant women living in the Raval district (Barcelona). They bring along their religions and traditions and enrich culturally this zone of the city. In this paper, we have researched the role new technologies have in the lives of these women coming from very different religions and countries. Our goal is to unveil if these digital tools could help them to achieve gender equality and the integration into their new country. To accomplish that, we have interviewed 238 women and have gathered relevant information. We have realized that although they have access to new technologies, they do not use them for equality or integration. Technology is, for immigrant women in Raval, a tool that connects them to their origins and countries more than a tool for integration in the new context.
\end{abstract}

Keywords: Woman, equality, religion, technology, social media, immigration, gender. 


\section{Introducción}

El barrio del Raval de Barcelona (Cataluña, España) acoge a 48.485 habitantes. Un 45,7\% son personas inmigrantes. 22.068 son mujeres. Más allá de las cifras, este distrito de la capital catalana se caracteriza por ser un núcleo rico en diversidad de culturas, lenguas, religiones y escenario de costumbres y tradiciones procedentes de todos los rincones del mundo. Esta diversidad cultural y religiosa del Raval se encuentra en un entorno - Cataluña- en el que un $52,4 \%$ de los habitantes se considera católico y donde en julio de 2015 se aprobó una ley que promulga que el derecho fundamental de igualdad entre mujeres y hombres se ejerza de forma efectiva en los ámbitos político, económico, social y cultural.

La convivencia y vínculo de estas dos realidades son de especial interés para el estudio que presentamos. En él, ponemos en el centro a las mujeres inmigrantes de distintas confesiones y tradiciones religiosas que viven en el barrio del Raval con el objetivo de saber cuál es su vínculo con las nuevas tecnologías. Analizamos si tienen el mismo acceso a ellas que los hombres, para qué las utilizan y si son una herramienta que promueve integración y la igualdad.

Estudios previos han demostrado que el nexo entre mujer, inmigración y tecnología es un campo de estudio que se expande. En "Discurso, género e inmigración", Enrique Santamaría hace referencia a los discursos sobre la inmigración no comunitaria donde insiste que hay una elipsis en el conocimiento de las reflexiones y luchas específicas que las mujeres que han emigrado llevan a cabo dentro de sus comunidades de pertenencia. (Santamaría, 1997: 40).

En su estudio sobre la participación de la mujer española en la vida social, económica y profesional, Mally y Orlando (2015) sitúan la cuestión del rol de la mujer en la vida pública y muestran la mejoría desde los años 50, pero siguen señalando como la ratio en comparación con los estándares europeos es baja, y no se refieren en concreto a la situación de la mujer inmigrante, mucho más vulnerable a esta socialización. (Stella-Orlando, 2015: 31).

Cabe destacar, en este sentido, que según el último informe realizado por la Fundación Mobile World Capital Barcelona (2016) sobre la brecha digital en la ciudad, existe una diferencia notable entre las actividades relacionadas con la tecnología realizadas por parte de hombres y las que llevan a cabo las mujeres. Las cifras correspondientes a toda la ciudad en general no muestran una brecha digital; en cambio, sí que lo hacen los resultados obtenidos en los barrios con una renta baja, en los que viven hombres y mujeres llegados de muchos países, como es el caso del Raval.

En el momento actual, además, las nuevas tecnologías y los dispositivos móviles se han puesto al alcance de la mayoría de la población y su uso ya se traduce en un valor mucho más que tecnológico, también social (Traxler, 2015). De hecho, esta situación representa lo que ya identificaron autores como McLuhan (1962): los avances tecnológicos comportan cambios sociales. 


\section{Metodología}

Ante la situación, y con el objetivo de responder a las cuestiones planteadas, hemos realizado un estudio de una duración de seis meses que se ha basado en dos herramientas metodológicas: la encuesta y la entrevista. Así, desde el mes de mayo hasta noviembre del 2015, hemos entrevistado a un total de 238 mujeres inmigrantes de distintos perfiles y confesiones de la zona del Raval.

Se ha llegado a ellas a través de las organizaciones o entidades del distrito que trabajan con el perfil analizado como público. Las entidades escogidas se han seleccionado por la relevancia, el valor social de su tarea así como por el alcance de esta. Igualmente, la diversidad de perfiles ha sido un criterio a tener en cuenta en el momento de escoger las organizaciones a través de las que contactar con las mujeres. Associació Consciència Krishna, Federació d'Entitats Cíviques i Religioses Filipines a Barcelona, Lloc de la Dona, Obra Missionera Ekumene, Església Evangèlica Protestant Barcelona Centre, Grup Interreligiós del Raval, Comunitat de l'Anyell, Comunitat Sant'Egidio i Centre Islàmic Cultural de Catalunya son las entidades que han colaborado en la investigación.

Por un lado, la elección de la encuesta se justifica por el objetivo de llegar a un alcance de respuestas representativo que poder valorar (Kerlinger, 1986) y equilibrado así como de obtener información con la suficiente sinceridad. Se trata de poder analizar y valorar datos comparables de una forma rápida, sistemática y eficaz (De Vaus, 2014). Cabe destacar, sin embargo, que, al realizar un cuestionario de respuestas cerradas, la sinceridad obtenida es óptima pero no total (Busquet, Medina i Sort, 2006). A pesar de ello, las participantes contaban con la posibilidad de ampliar la información en un apartado final destinado a comentarios. La citada encuesta nos ha permitido obtener resultados cuantitativos que hemos podido resumir en los datos concretos, detallados en el presente artículo.

El cuestionario realizado se estructura en seis categorías, que nos han permitido agrupar también las respuestas obtenidas y centrar nuestro análisis en los distintos aspectos que nos hemos propuesto descubrir. Los seis citados grupos son:

- Información general: para conocer su perfil y recabar datos que nos permitieran situar demográficamente y socialmente a las personas encuestadas. Es por eso que los campos principales del apartado son: nombre, edad, estado civil, número de hijos, localidad, estudios, procedencia, religión, profesión, tiempo viviendo en Barcelona y email.

- Internet: para estudiar el nivel de uso y accesibilidad de las plataformas online por parte de estas mujeres. En este sentido, les hemos preguntado acerca de la accesibilidad de internet en el hogar, la frecuencia de uso, el motivo del consumo, las redes en las que tienen perfil, el uso de internet en religión, así como el uso del correo electrónico. 
- Redes sociales: se trata de conocer si las mujeres tienen actividad y perfiles en espacios digitales sociales como Twitter o Facebook. En este caso, los campos a contestar hacen referencia al nivel de anonimato de sus cuentas, a la procedencia de sus contactos y a la opinión de las mujeres sobre la seguridad de estos espacios. Además, se cuestiona también si la usuaria se considera activa o pasiva.

- Teléfono móvil o smartphone: como en el caso anterior, con el objetivo de saber igualmente, en qué medida las mujeres encuestadas tienen acceso a él, para qué actividades lo utilizan y en qué lengua las llevan a cabo.

- Opinión: más allá del consumo que realizan de estos espacios, se trata de saber qué piensan de la actividad que se desarrolla en ellos y de cómo ven la imagen que se da de su religión y cultura. Es por eso que el cuestionario se cierra con nueve afirmaciones; algunas sobre la visión que dan los medios de la mujer inmigrante y otras sobre la comodidad y habilidad de uso de las nuevas tecnologías que las mujeres encuestadas se reconocen a ellas mismas.

En total, se han distribuido 300 cuestionarios de los cuales hemos obtenido 238 respuestas. Estas 238 se han realizado mediante la encuesta en papel y de forma presencial. En la mayoría de casos, han tenido lugar en las sedes de las entidades que acogen a las mujeres encuestadas de las distintas confesiones. En todos los casos, ésta ha sido la preferencia de la entidad contactada.

La técnica de la encuesta la avalan otros estudios que, por temática o por ámbito, son cercanos al presente. En este sentido, encontramos ejemplos como:

- La brecha digital de género en la juventud española (2010), investigación realizada por la Universidad Complutense de Madrid en el marco del proyecto e-Igualdad.net. En este caso, los autores realizaron una encuesta online a 400 alumnos de diez institutos de cinco comunidades autónomas para analizar la existencia de una posible brecha digital de género entre los jóvenes.

- Informe Jóvenes 2000 y religión (2002), estudio realizado por la Fundación Santa María con un cuestionario a 1.072 adolescentes de todo el país. El objetivo, conocer el papel y la influencia de la religión en las nuevas generaciones.

- Medios de comunicación y religión en España. Una investigación sobre el estado de la comunicación mediática Iglesia-sociedad.(2005). Editado también por la Fundación Santa María y llevado a cabo por la Universidad Pontificia Comillas de Madrid y el Instituto Diocesano de Teología y Pastoral de la Diócesis de Bilbao, este análisis tiene el objetivo 
de identificar la opinión de los actores implicados en la comunicación Iglesia-mediossociedad sobre el estado de la comunicación mediática Iglesia-sociedad. Utiliza la encuesta como método tanto cuantitativo como cualitativo.

Con las citadas encuestas, hemos identificado diversos perfiles. Cabe detallar que el $50 \%$ de las mujeres consultadas están casadas, el 79\% tiene hijos y el 43\% se sitúa en la franja de edad de entre los 30 y los 49 años. De entre ellas, el 6\% no tiene formación, el 15\% cuenta con estudios de educación primaria y el $31 \%$ ha realizado formación equivalente a la educación secundaria. Proceden de países como Filipinas, India, Pakistán, Bulgaria, Camerún, Nigeria, Mali, Argelia, Marruecos, Chile, Argentina, Bolivia, Perú, Ecuador o Colombia. Cuanto a su religión, en la mayoría de los casos se trata de mujeres musulmanas, pertenecientes a la diversas denominaciones cristianas o bien a la comunidad Hare Krishna.

Se trata de un perfil muy concreto pero abundante en la zona. Muchas aseguran que sus historias aparecen en los medios con menos frecuencia que las de los hombres de su mismo perfil. En este caso, se trata de darles visibilidad y de conocer cómo se traduce, en su entorno, la continua e imparable irrupción de la tecnología en la vida diaria.

La otra técnica metodológica utilizada en esta investigación es la entrevista, que nos ha servido para complementar y reforzar los datos recabados. En este caso, nos permite obtener ideas, reflexiones así como nuevas dimensiones de la temática tratada (Busquet, Medina y Sort, 2006). Las entrevistas se han realizado, por un lado, a cinco mujeres representantes de las distintas comunidades contactadas. De éstas se ha elaborado un vídeo resumen publicado en el portal Women Tech Religion ${ }^{1}$. Por otro lado, también hemos entrevistado a Louise Guido (Febrero 2016), experta en la materia, fundadora de Smart Woman, una plataforma online y móvil que tiene el objetivo de empoderar a la mujeres de todo el mundo mediante la difusión de conocimiento y la creación de comunidades online. Guido encabeza además, la compañía ChangeCorp, líder global en la creación de servicios móviles innovadores en mercados emergentes, en los que trabaja e interviene de forma directa. Además, está detrás de la Foundation for Social Change, una entidad sin ánimo de lucro dedicada a enseñar y formar profesional y personalmente a chicas y mujeres en países emergentes; en muchos casos, a través de las nuevas tecnologías.

La entrevista es también una técnica utilizada en investigaciones previas y similares en temática. Un ejemplo es el informe The Status of Women in the U.S. Media 2015, producido por la organización Women's Media Center de Estados Unidos. El ya citado estudio, 'Medios de comunicación y religión en España. Una investigación sobre el estado de la comunicación mediática Iglesia-sociedad', también utiliza la entrevista.

Nuestro análisis parte del proyecto "Digital Narratives. Gender, Immigration and Religion on the Move", liderado por el Observatorio Blanquerna de Comunicación, Religión y Cultura y

${ }^{1}$ [en línea] Disponible en: http://www.womentechreligion.com/ [25/01/2016]. 
financiado por la World Association for Christian Communication a través de Iglesia Valdense y su programa Otto per Mille.

\section{Accesibilidad e igualdad}

Según Louise Guido (2016), las nuevas tecnologías proveen a las mujeres de una vía de comunicación propia que les permite tener contacto con otras mujeres en su misma situación fuera de su entorno más inmediato.

En este sentido, la accesibilidad de las mujeres a la tecnología móvil es un elemento clave. Del total de encuestadas, un $74 \%$ dispone de internet en casa mientras un $24 \%$ no tiene acceso a él. Disponibilidad sin embargo, no siempre es sinónimo de accesibilidad.

Debemos tener en cuenta que, en bastantes casos, es la misma tradición la que impone a los hombres actividades más vinculadas con la independencia o la comunicación fuera del entorno más próximo. Las mujeres inmigrantes, muchas veces, son las principales garantes y mantenedoras de las tradiciones en algunas de estas familias (Roque, 1999) así como de los preceptos de las religiones cuanto a temas como la sexualidad o la familia. Cabe recordar que no sólo son las religiones como la musulmana las que expresan aspectos concretos a cumplir en estos asuntos, también la tradición católica y judeocristiana lo hace (Del Valle y Sanz Rueda, 1991).

Si nos fijamos en el ejemplo de núcleos familiares llegados de Marruecos, Roque (1999) plantea dos tipologías de familia a tener en cuenta: tradicional -patrilineal y endógama- y moderna, de carácter nuclear. En esta citada tipología clásica y tradicional, se define una comunidad cerrada en el que los lazos son muy fuertes pero en las que las relaciones entre las mujeres están destinadas a romperse, pues su tarea se centra en dedicarse a la familia y a su marido.

En este sentido, debemos poner sobre la mesa la duplicidad de referentes que, una vez en su nuevo entorno, las mujeres inmigrantes tienen: los del mundo exterior y los de su entorno 'interior'. Aunque se crea muchas veces, los exteriores no siempre modifican a los interiores. Así se muestra en algunos estudios sobre la inmigración femenina en Europa (Ribas, 1999). En ellos se destaca que muchas familias inmigrantes tienden a reproducir los gustos consumistas occidentales o, en el caso de los magrebíes, desdibujar la fe en el islam. Estos estudios muestran también la visión crítica de estas comunidades sobre este fenómeno y descubren un perfil de inmigrante que no asimila íntegramente su nuevo entorno y que conserva, e incluso refuerza, sus raíces.

Cuanto a la mujer, Guido (2016) destaca la importancia del concepto de la comunidad en el camino hacia la igualdad de género. Las nuevas tecnologías aparecen como el espacio 
privado y propio de las mujeres para que los lazos con otras no se rompan; las redes virtuales de mujeres contribuyen a empoderarlas (Youngs, 2004), y no sólo en la red y en el espacio digital.

Chambers, Steiner y Fleming (2004) ya plantean que internet impulsa el discurso público y fortalece la esfera pública a través de una lógica de comunidad:

"The internet has produced virtual communities linked by social or community issues and is contributing broader and more in-depth political participation by citizens. [...] Interactive electronic technology offers the potential to empower citizens to take part in new democratic debates among one another and with governments" (Chambers, Steiner y Fleming, 2004: 239).

La creación y consolidación de estas redes que contribuyen al empoderamiento y a la igualdad en el caso de las mujeres inmigrantes del Raval va más allá del acceso o la disponibilidad de conexión a internet en casa. El acceso a nuevas tecnologías y la igualdad en este aspecto también implica la posesión, por parte de las mujeres, de dispositivos y sistemas propios con conexión a la red.

Los datos obtenidos, en este sentido, indican que las mujeres inmigrantes del barrio del Raval tienen, en un $88 \%$ de los casos, un teléfono móvil propio, aunque sólo el $56 \%$ tiene un dispositivo con acceso a internet o smartphone. En cambio, si nos fijamos en la cifra de mujeres que disponen de tabletas propias, se reduce al $29 \%$.

Según Guido (2016), en los países en los que realiza intervención directa, existen muchas situaciones en las que las mujeres no tienen teléfono propio por deseo de sus maridos, padres o hermanos. En algunos además, disponer de este elemento tecnológico, considerado de valor, es un derecho que los hombres piensan que les corresponde.

Un ejemplo claro de la situación lo muestra el proyecto de aplicación móvil Zero Mothers Die. La iniciativa, de la empresa catalana Universal Doctor, provee a mujeres embarazadas de comunidades, generalmente africanas, de dispositivos móviles para realizarles un seguimiento más cercano de la gestación y evitar la mortalidad materna. Sin embargo, los primeros dispositivos entregados acabaron en manos de los hombres; la iniciativa da ahora a las mujeres teléfonos móviles de color de rosa, de modo que los maridos, hermanos o padres no se apropian de ellos por su estética.

Por otra parte, analizamos también el acceso de las mujeres encuestadas a las redes sociales.

En este sentido, descubrimos que tienen, en la mayoría de los casos, perfil en dos espacios concretos, Facebook y Skype, las redes que más conocen. A pesar de ello, sólo el 24\% de las 238 mujeres encuestadas tiene perfil en Facebook y 67\% habla vía la plataforma Skype. Las demás redes no están, en general, en el entorno habitual de las mujeres inmigrantes del barrio del Raval y son pocas las que cuentan con perfil en You Tube o Instagram. Cuanto a Twitter, un 95\% de las mujeres encuestadas no está presente ni dispone de perfil. 
La limitada y desdibujada presencia digital en redes sociales de las mujeres que nos indica la muestra analizada nos hace pensar que, a pesar de que las herramientas están disponibles, la falta de acceso a ellas retrasa el empoderamiento de la mujer y a la vez, alarga el camino hacia la igualdad de género.

El concepto de comunidad remarcado por Guido (2016) y también por Youngs (2004) -una comunidad que las mujeres pueden construir mediante estas vías- se ve todavía limitado por la falta de uso -acceso, en su mayoría, tienen-, sea o no por voluntad propia, a estos espacios de diálogo y de posible integración.

\section{Familiaridad y confianza en las nuevas tecnologías}

La falta de uso, y en algunos casos, de acceso a las nuevas tecnologías, no es el único motivo que limita la construcción de comunidad y la igualdad digital de las mujeres inmigrantes en el barrio del Raval.

En las entrevistas realizadas detectamos que, en todos los aspectos, existen conceptos vinculados a las nuevas tecnologías que las mujeres inmigrantes del Raval no entienden y hace que no puedan responder a algunas de las cuestiones realizadas sobre, por ejemplo, páginas web o redes sociales. Nos planteamos pues, hasta qué punto la disponibilidad y el acceso a las nuevas tecnologías comporta la habilidad de hacer un uso óptimo de ellas.

Los datos señalan que todavía son muchas las mujeres inmigrantes en el barrio del Raval que no cuentan con la formación necesaria para utilizar el ordenador, el teléfono móvil o la tableta aprovechando todas las posibilidades que estos dispositivos ofrecen.

De hecho, cuando planteamos a las mujeres encuestadas si utilizan internet, un $12 \%$ asegura que no lo sabe; un 9\% no puede responder si tiene tableta, un 39\% no sabe si dispone de un blog personal, el 38\% explica no saber si tiene web y el $36 \%$ no entiende los conceptos relacionados con las redes sociales.

Las cifras muestran pues, que un $31 \%$ de las mujeres inmigrantes con las que hemos hablado admite no tener el suficiente conocimiento sobre las nuevas tecnologías y sobre cómo utilizarlas. El 38\% asegura no saber si tiene este conocimiento.

Esta realidad contrasta con el planteamiento teórico que Sadie Plant (1997) realiza cuando explica que las mujeres están mejor preparadas, cultural y psicológicamente, para el siglo XXI y que se desarrollan mejor que los hombres en el espacio digital. Cabe argumentar pues, que es posible que esta realidad se de actualmente pero nuestro estudio muestra que no en todos los ámbitos de la sociedad.

Si las nuevas tecnologías son una herramienta para el empoderamiento y la igualdad de género, que exista una brecha de formación en el uso de ellas en el caso de las mujeres 
inmigrantes es una barrera a saltar. Esta brecha, ya detectada en el informe de la Fundación Mobile World Capital sobre la brecha digital en Barcelona (2016), se confirma en el barrio del Raval.

Por otro lado, y también a pesar de la elevada accesibilidad mostrada anteriormente, cuando nos fijamos en la frecuencia de uso de las nuevas tecnologías sólo 136 de las 238 mujeres encuestadas aseguran que es diaria.

Además, haciendo hincapié en las actividades que realizan estas mujeres a través de las nuevas tecnologías, destacan datos como que un $31 \%$ explica que no usa internet para comunicarse con otras personas. De hecho, todavía es de un $21 \%$ el total de las mujeres que no llama desde un teléfono móvil sino desde una cabina o locutorio.

Más allá de la familiaridad o de la formación de estas mujeres en el uso de las nuevas tecnologías, la opinión y la confianza que les merecen es una cuestión también objeto de este estudio. En este sentido, cabe destacar que el $48 \%$ de las mujeres que utilizan redes sociales lo hace con perfiles que están a su nombre, pero un $15 \%$ utiliza seudónimos. Además, un $10 \%$ de ellas no tiene una opinión positiva de las redes sociales y un $30 \%$ no cree que el medio digital sea su mejor vía de expresión.

Cabe añadir que el estudio también nos ha llevado a conocer, más allá de la visibilidad digital de las mujeres encuestadas, la opinión que tienen de su presencia y la de su cultura y religión en los medios. La posibilidad de que se genere desconfianza en el medio digital y que muchas no crean que éste sea su mejor medio de expresión, ¿podría venir generada por el trato recibido por parte de los medios tradicionales?

El 36\% de las mujeres con las que hemos hablado desde el Observatorio piensa que los medios ignoran a la mujer creyente e inmigrante en su situación; el 45\% no tiene respuesta para esta cuestión mientras sólo un $20 \%$ cree que su perfil tiene presencia en los medios de comunicación.

Al plantear si los citados medios tratan los temas y asuntos que incumben a estas mujeres, un $18 \%$ cree que no, el $45 \%$ no responde y el $36 \%$ piensa, en este caso, que sí. En este sentido, además, y poniendo sobre la mesa si los medios muestran una imagen negativa de la mujer inmigrante en la situación de las que hemos entrevistado, el 21\% piensa que sí; el 50\% asegura no tener respuesta a esta pregunta mientras un $29 \%$ cree que no es así, es decir, que la imagen que dan es positiva.

La representación de la mujer -independientemente del país del que provenga- en los medios de comunicación no es un factor baladí. Según López Díez (2004) - visión compartida por estudios feministas de los medios, como el de Van Zoonen (1994)- la mirada con la que se ha construido la realidad hasta el momento desde los medios ha sido androcéntrica y realizada desde quien ha detentado tradicionalmente el poder. Añade, que las representaciones que se realizan en los medios masivos no son inocentes, sino que se desarrollan en el marco de las relaciones de poder. En este mismo sentido, afirma Stuart Hall (1997): 
"Es a través del uso que hacemos de las cosas, y de lo que decimos, pensamos y sentimos acerca de ellas -cómo las representamos- como les damos un significado. En parte, damos significado a las cosas a través de cómo las usamos o las integramos en nuestras prácticas diarias. Es nuestro uso de una pila de ladrillos y de cemento lo que los hace una 'casa'; y lo que sentimos, pensamos o decimos acerca de ella lo que hace a una 'casa' un 'hogar'. En parte, damos significado a las cosas a través de cómo las representamos -las palabras que usamos para referirnos a ellas, las historias que contamos sobre ellas, las imágenes que producimos de ellas, las emociones que asociamos con ellas, las maneras en cómo las clasificamos y conceptualizamos, los valores que asociamos a ellas" (Hall, 1997:3).

Detectamos pues, en la representación y limitada presencia de las mujeres inmigrantes en los medios online, la posible existencia de un cierto legado de la tradicional representación femenina en los medios tradicionales, y podemos deducir que el tipo de representación expresada por López Díez (2004) y Stuart Hall (1997) ha creado un cierto patrón que puede tener algún tipo de influencia en la discreta presencia de muchas mujeres -especialmente perfiles como los entrevistados, fieles a la tradición y a muchos valores religiosos- en el espacio digital. "The internet reflects, and in some way extends, traditional patterns of power and oppression, as well as opening up opportunities for new forms of politics, collaboration and contestation" (Youngs, 2004: 196).

Cabe añadir que los estudios sobre representación y presencia de la mujer así como de tratamiento del género no sólo analizan esta representación tradicional, también la falta de presencia femenina implícita y explícita en muchos casos; desde la imagen, hasta en los textos o en la iconografía (Bach Arús et al., 2000). Análisis de autores como Vigara Tauste y Jiménez Catalán (2002) refuerzan esta tesis.

\section{Tecnología, integración y religión}

¿Es la tecnología una herramienta para promover la integración de las mujeres en las nuevas sociedades que las acogen? Louise Guido (2016) responde un contundente sí a esta pregunta y pone como ejemplo las mujeres que llegan al centro de Europa desde países como Siria. Guido (2016) asegura que la gran mayoría dispone de teléfono móvil.

Si nos fijamos en cuál es el uso mayoritario que las mujeres encuestadas hacen de las nuevas tecnologías es comunicarse con los demás. Sin embargo, detectamos en los resultados de las encuestas que, en el caso de las mujeres inmigrantes que viven en el barrio intercultural del Raval de Barcelona, la tecnología sirve para comunicarse y para crear la comunidad 
mencionada pero, en la mayoría de los casos, no para impulsar y promover la integración con otras mujeres de la ciudad o inmigrantes de otros países.

Las mujeres inmigrantes que utilizan nuevas tecnologías en el barrio del Raval lo hacen para mantenerse arraigadas y conectadas a la comunidad que ya tenían creada presencialmente en sus países maternos. Nos lo muestran los datos; sólo el $14 \%$ de las mujeres encuestadas asegura utilizar las redes sociales para comunicarse con personas que no procedan de su país de origen. De hecho, el $32 \%$ admite que utiliza las redes sociales para hablar con su familia y otro porcentaje elevado (un 26\%) asegura que las redes le suponen una buena herramienta para mantener el contacto con las personas que viven en su país de origen.

Además, si tenemos en cuenta también que el $31 \%$ utiliza la tecnología en su lengua materna y no en catalán o castellano, vemos que el potencial de integración e igualdad de estos recursos no se acaba de aprovechar del todo.

Las redes, y el espacio digital en general, son un 'no lugar' (Augé,1994) en el que no existen fronteras. Tal como asegura Youngs (2004):

"Virtual space has been understood partly in "frontier" terms. It represents not just an additional social arena, or set of interrelating spaces, but a sphere, which in its nature sharply contrasts with other traditional social spheres. These traditional settings are physical, often clearly bounded locations or places -the home, the nation, the city, the council or parliament chamber, etc. The internet represents a new frontier in transcending, in significant senses, many of the physical constraints of, and boundaries between such traditional settings. The internet is characterized by its cross boundary nature, and in this way and others goes beyond the (physical and geographical) world we have previously inhabited" (Youngs, 2004: 188).

Por lo tanto, para las mujeres llegadas a un nuevo entorno pueden ser un elemento reconfortante y de conexión con sus lugares de origen. En su caso, sin embargo, no se convierte en un espacio de encuentro con personas de su nuevo entorno y en el que puedan obtener nuevos conocimientos o practicar una nueva lengua.

Estamos pues, en un espacio de libertad absoluta pero que a la vez, encierra a las mujeres que no se abren a su propio entorno, con lo cual, descubrimos una 'falsa libertad'. Los usos que las mujeres dan a las nuevas tecnologías, según la información recabada, son significativos en este sentido.

Cabe reflexionar, en este punto, sobre los usos femeninos de las nuevas tecnologías desde la perspectiva de la 'esfera pública' de Habermas (1989) - que corresponde al espacio para la libre deliberación política y la participación en el debate y la crítica de otras esferas, como el gobierno, la empresa o la familia-. Muchos estudios (Benhabib, 1992) han revisado críticamente el concepto por estar formulado, inicialmente, ignorando el género y la diferencia de géneros. Según Chambers, Steiner y Fleming (2004) y Youngs (2004) el concepto excluye a la mujer 
porque ella siempre ha funcionado como apoyo a la actividad de los hombres como ciudadanos libres e iguales, actuando desde una 'esfera privada'. Para Van Zoonen (1991), la mujer ha sido históricamente parte de esta 'esfera pública', pero su contribución ha sido obstaculizada, ignorada y marginada.

Es Rita Felski (1989) quien elabora un discurso opuesto a Habermas (1989) y desarrolla una alternativa feminista al modelo de 'esfera pública'. En este sentido, la producción y circulación de nuevos medios alternativos para mujeres genera y confirma la identidad femenina y la noción de comunidad entre mujeres (Chambers, Steiner y Fleming, 2004). Las autoras también plantean si, a pesar de que esta noción se desarrolla sobre la base de la prensa escrita, internet puede convertirse en un espacio de expresión democrático para la mujer: "Certainly cyberfeminists believe that a feminist counter-public can flourish in cyberspace, and that new communication technologies are liberatory" (Chambers, Steiner y Fleming, 2004: 239).

Guido (2016) y Roque (1999) coinciden en que las mujeres son las que mantienen principalmente la esencia de la tradición, la religión y la cultura en los núcleos familiares, sobre todo en el caso de familias que llegan a nuevos países. Suponen pues, el perfil de inmigrante conservador que detallaba Ribas (1999).

La religión es un elemento de suma importancia para ellas, y la actividad en la red -de las que la realizan- lo muestra. De hecho, el $51 \%$ de las mujeres encuestadas asegura hacer búsquedas en internet relacionadas con la religión. Un 39\%, además admite sólo comunicarse en redes sociales con personas de su misma religión, a pesar de que el $75 \%$ explica que se encuentra en redes con personas de otras religiones. Encontrarse, sin embargo, no siempre comporta tener contacto.

El estudio muestra que, de momento, en el caso de las mujeres del barrio del Raval de Barcelona, las nuevas tecnologías no contribuyen tampoco al contacto con otras religiones. Alguna de las mujeres afirma tajante lo que anuncian Roque (1999) y Guido (2016): muchas mujeres se mantienen fieles a las tradiciones y a las religiones y de hecho, en algún caso expresan que religión "sólo existe una" y se muestran escépticas a saber más sobre ella en la red porque explican que ya la han aprendido y conocido durante toda su vida.

Cabe añadir que existen más de un millón de páginas sobre religión en internet (Duteil Ogata et al., 2015) y se trata de un ámbito que según muchos autores se ha adaptado rápidamente a las nuevas tecnologías hasta en formato (Kolodziejska, 2015); ha sido, en algunos aspectos pionero.

En este punto, es relevante analizar el estudio realizado por Mia Lövheim (2014), 'Une voix a elles: jeunes, musulmanes et blogueuses'. En él, estudia tres blogs y la actividad en redes sociales de tres jóvenes musulmanas que viven en países escandinavos. La investigación muestra el potencial visibilizador e integrador de las citadas herramientas, no siempre utilizado en el caso del Raval. 
“Malgré leurs différences, ces trois blogueuses représentent des exemples de la manière dont les jeunes femmes se sont mises à utiliser les nouveaux médias, en particulier les blogs, de manière à participer à un sphère publique médiatisée et à agir comme des agents indépendants capables de se lancer dans le débat religieux depuis une position d'autorité" (Lövheim, 2014: 318).

Este estudio pues, no hace sino reforzar lo citados planteamientos de Chambers, Steiner y Fleming (2004) sobre internet como un espacio de expresión para la mujer y como una herramienta que contribuye a la igualdad.

Vemos, sin embargo, que en este aspecto, la realidad y los planteamientos son todavía dispares. Sobre todo, teniendo en cuenta los distintos espacios sociales y situaciones en los que se encuentran las mujeres.

\section{Conclusiones}

El estudio mostrado nos ha llevado a reflexionar sobre distintos aspectos vinculados con los temas que nos ocupan: género, igualdad, integración, nuevas tecnologías y religión.

El análisis de las entrevistas realizadas complementado con la perspectiva teórica de la temática estudiada nos ha permitido elaborar una comparación que muestra en qué punto, momento y situación se encuentran las mujeres inmigrantes del Raval cuanto a gestión de las nuevas tecnologías en el camino hacia la igualdad. Este doble punto de vista nos ha llevado a desvelar la realidad del distrito y a partir de aquí, nuestra investigación se resume en las conclusiones que exponemos a continuación.

En primer lugar, detectamos que las herramientas digitales son un elemento que puede contribuir a la integración y a la igualdad de género por la creación de una comunidad más allá del entorno próximo de las mujeres inmigrantes que viven en el barrio del Raval.

Son una vía para la creación de redes que van más allá de las relaciones que de forma directa las mujeres pueden tener con su entorno más inmediato. Autores como Youngs (2004) o Chambers, Steiner y Fleming (2004) destacan cómo el entorno digital supone un espacio que amplía la 'esfera pública' de Habermas (1989) y la lleva más allá del hombre; la pone al alcance de las mujeres, que representadas por los medios habitualmente en situaciones y posiciones tradicionales (Van Zoonen, 1994), habían contribuido desde una discreta y secundaria posición a la 'esfera pública'.

De este modo, observamos el espacio digital y las herramientas que de él se derivan como una importante vía para favorecer la igualdad de género. Las herramientas digitales dan a las mujeres inmigrantes una oportunidad de suma importancia: expresar su opinión e intereses así como todos los aspectos que les inquietan y que forman parte de su vida diaria. 
En el caso de las mujeres encuestadas, el acceso a este nuevo espacio, que está generalmente expandido, supone también un camino hacia la integración en la nueva sociedad en la que conviven. El caso del Raval es además, excepcional. La cantidad de culturas que acoge es muy elevada, pero vías de comunicación y encuentro entre ellas pueden facilitar la creación de nuevas relaciones interculturales e interreligiosas. Recordemos que un elevado porcentaje de las mujeres encuestadas (el 75\%) afirma encontrarse en redes con personas que no son de su misma religión.

Sin embargo, y a pesar de las grandes posibilidades que vemos que estos medios ofrecen, las nuevas tecnologías no se utilizan, en el caso de las mujeres creyentes inmigrantes del Raval, de una forma totalmente óptima como para conseguir los citado hitos y aprovechar toda su potencialidad.

El estudio nos ha permitido conocer sus hábitos de consumo y de acceso a las nuevas tecnologías así como sus impresiones y opiniones sobre ellas. Hemos visto que, por el momento, son todavía muchas las mujeres que no utilizan las nuevas tecnologías (ya sea ordenador, dispositivos móviles, redes sociales) en su día a día y para comunicarse con otras mujeres de su nuevo entorno. Por lo tanto, las comunidades que podrían crearse no están del todo desarrolladas ni consolidadas.

Nos hemos dado cuenta de que las mujeres que utilizan las nuevas tecnologías y las redes sociales no lo hacen tanto para abrirse a su nueva sociedad como para mantenerse arraigadas a sus raíces. La comunicación con personas de su entorno en su país es una de las actividades para las que más se sirven de las nuevas tecnologías. Además, la mayoría se expresa en estas plataformas en su lengua materna, con lo cual, tampoco se convierten en una herramienta para aprender y ganar fluidez en catalán o castellano.

La falta de formación en el uso de estas nuevas tecnologías es otro de los motivos que hemos descubierto que influye en esta situación. Como hemos remarcado, muchas de las mujeres encuestadas no han comprendido conceptos surgidos de plataformas o herramientas digitales; este hecho muestra que tener acceso a ellas no significa saber utilizarlas adecuadamente y poder aprovechar todas las ventajas que ofrecen.

Finalmente, hemos resuelto también que la religión y las tradiciones son elementos que pueden adaptarse - y de hecho lo están- al espacio digital pero que, en el caso de muchas mujeres inmigrantes el Raval, suponen todavía un freno para entrar en el mundo 2.0.

Resta descubrir en futuros análisis sobre la temática aspectos como el interés o la inquietud de estas mujeres en obtener la formación necesaria para utilizar estas nuevas herramientas; ver si la situación es similar o divergente en otras zonas de la ciudad de Barcelona o incluso en otras localidades, provincias o países. 


\section{BIBLIOGRAFÍA}

- Ajuntament de Barcelona (2015): "La població estrangera a Barcelona", [en línea] Disponible en: http://www.bcn.cat/estadistica/catala/dades/inf/pobest/pobest15/pobest15.pdf [27/02/2016]

- Augé, Marc (1994): “Los 'no lugares'. Espacios del anonimato. Una antropología de la sobremodernidad”. En: Estudios Geográficos no. 5, pp. 40-83.

- Bach Arús, Marta et al. (2000): El sexo es noticia. Reflexiones sobre el género en la información y recomendaciones de estilo. Barcelona: Icaria. Sociedad y opinión.

- Benhabib, Seyla (1992): "Models of public space: Hannah Arendt, the liberal tradition, and Jürgen Habermas". En: Craig Calhoun (1992) (ed.): Habermas and the public sphere. Cambridge: MIT Press, pp. 73-98.

- Busquet, Jordi; Medina, Alfons; Sort, Josep (2006): La recerca en comunicació. Barcelona: Editorial UOC.

- Chambers, Deborah; Steiner, Linda y Fleming, Carole (2004): Women and Journalism. London: Routledge.

- De Vaus, David. (2014): Surveys in social research. New York: Routledge.

- Del Valle, Teresa; Sanz Rueda, Carmela. (1991) Género y sexualidad. Madrid: Universidad Nacional de Educación a distancia. Fundación Universidad Empresa.

- Douyère David et al. (2014) (dir.): Religion \& Communication. Paris: L’Harmattan.

- Duteil-Ogata, Fabienne; Jonveaux, Isabelle; Kuczynski, Liliane; Nizard, Sophie (2015): (dir.): Le religieux sur Internet. Paris: L'Harmattan.

- Elzo, Javier (dir.) (2002): Jóvenes 2000 y religión. Madrid: Fundación Santa María.

- Felski, Rita (1989): Beyond feminist aesthetics: feminist literature and social change. Cambridge: Harvard University Press.

- Fraser, Nancy (1992): "Rethinking the public sphere: a contribution to the critique of actually existing democracy". En: Craig Calhoun (ed.): Habermas and the public sphere. Cambridge: MIT Press, pp. 42-109

- Fundació Mobile World Capital Barcelona (2016): "L'escletxa digital a la ciutat de Barcelona", [en línea] Disponible en: http://www.mobileworldcapital.com/escletxadigital/\#5thPage/1 [27/02/2016].

- Fundació Tot Raval (2011): “Estudi econòmic i comercial del Raval 2010-2011”, [en línea] Disponible en: http://www.totraval.org/publicacion/23/estudi-economic-i-comercial-del-raval2010-2011 [27/02/2016].

- Guido, Louise M. [Entrevista], Barcelona, 25-02-2016.

- Habermas, Jürgen (1989): The structural transformation of the public sphere: an inquiry into a category of bourgeois society. Cambridge: Polity Press.

- Hall, Stuart (1997): Representation: Cultural representations and signifying practices. London: Open University. 
- Kerlinger, Fred N. (1986): "Survey Research" En: Foundations of Behavioral Research in Education. New York: Holt, Rinehart, and Winston. pp. 599-611.

- Kolodziejska, Marta (2015): "How Catholic internet forums are changing Catholicism. The Polish experience”. En: Míriam Díez; Josep Lluís Micó y Josep Maria Carbonell (ed.): Catholic communities online. Barcelona: Facultat de Comunicació i Relacions Internacionals Blanquerna. pp. 83-94.

- López Díez, Pilar (2004) (dir.): Manual de información en género. Madrid: Instituto Oficial de Radio y Televisión.

- Lövheim, Mia (2015): “Une voix à elles: jeunes, musulmanes et blogueuses”. En: Fabienne Duteil-Ogata; Isabelle Jonveaux; Liliane Kuczynski y Sophie Nizard (dir.): Le religieux sur Internet. Paris: L'Harmattan, pp. 299-319.

- Mally, Stella - Orlando, Carlos (2015): Women's participation in economic, professional and social life. The case of Spain. Barcelona: Icaria Editorial.

- McLuhan, Marshall (1962): The Gutenberg Galaxy. Toronto: University of Toronto Press.

- Observatorio e-Igualdad (2010): "La brecha digital de género en la juventud española“, [en línea] Disponible en: http://eigualdad.net/sites/default/files/Brecha_digital_genero_juventud_espanola_2010.pdf [16/05/2016]

- Plant, Sadie (1997): Zeroes + Ones: Digital Women + The New Technoculture. London: Fourth Estate.

- Ribas, Natalia (1999): Las presencias de la inmigración femenina. Barcelona: Icaria Antrazyt.

- Roque, Maria-Àngels (1999) (dir.): Dona i migració a la Mediterrània occidental. Barcelona: Proa.

- Ross, Karen; Byerly, Carolyn M. (2004): Women and Media. Oxford: Blackwell Publishing.

- Santamaría, Enrique (1997): “Discurso, género e inmigración”. En: Problemas de Género, Archipiélag, nº. 30. Barcelona: Cuadernos de crítica y cultura, 1997, pp. 40-47.

- Traxler, John; Barcena, Elena; García Laborda, Jesús (2015): "Mobile technology for foreign language teaching: building bridges between non-formal and formal scenarios". En: Journal of Universal Computer Science, vol. 48, nº 1, pp. 1234-1247, [en línea] Disponible en: http://www.jucs.org/jucs_21_10/mobile_technology_for_foreign/jucs_21_10_1234_1247_edito rial.pdf [27/02/2016]

- Van Zoonen, Liesbet (1994): Feminist Media Studies. London: Sage Publications.

- Vidal Fernández, Fernando; González-Carvajal, Luis (2005): Medios de comunicación y religión en España. Madrid: Fundación Santa María.

- Vigara Tauste, Ana Má Jiménez Catalán, Rosa Ma (2002): 'Género', sexo, discurso. Madrid: Laberinto.

- Women's Media Center (2015): “The Status of Women in the U.S. Media 2015", [en línea] Disponible en: http://www.womensmediacenter.com/pages/2015-statistics [16/05/2016].

- Youngs, Gillian (2004): “Cyberspace: The New Feminist Frontier”. En: Karen Rossy Carolyn M. Byerly: Women and Media. Oxford: Blackwell Publishing, pp. 185-205. 